\title{
SEPARABILITY AND MINIMAL WEAK BASE TOPOLOGIES
}

\author{
S. W. DAVIS AND R. M. STEPHENSON, JR.
}

\begin{abstract}
In this note, we present filter characterizations of minimal weakly first countable, minimal $\mathscr{F}$, minimal $\mathscr{F}_{r}$, weakly first countableclosed, $\mathscr{F}$-closed, and $\mathscr{F}_{r}$-closed spaces. Our main result is a generalization of G. M. Reed's theorem that every Moore-closed space is separable.
\end{abstract}

0. Introduction. For a property $\mathbf{P}$ of topological spaces, we say a Hausdorff space with property $\mathbf{P}$ is $P$-closed provided that it is a closed subset of every Hausdorff space with property $P$ in which it can be embedded. A Hausdorff space $(X, \tau)$ with property $\mathrm{P}$ is called $P$-minimal if there is no Hausorff $P$-topology on $X$ which is strictly weaker than $\tau$.

The properties of $P$-closed and $P$-minimal spaces have been studied by numerous authors, e.g. see the reference list of $\left[\mathbf{S}_{1}\right]$ or $[\mathbf{C}]$. Recently, in $\left[\mathbf{S}_{2}\right], \mathbf{R}$. M. Stephenson, Jr. has characterized and studied symmetrizable-closed and minimal symmetrizable spaces. In this paper we present similar results concerning some of the spaces introduced in [A], [D], and [HS], namely $\mathscr{F}, \mathscr{F}_{r}$, and weakly first countable spaces.

Let us define these concepts.

Definition 0.1. A wfc-system for a topological space $X$ is a mapping $B$ : $\omega \times X \rightarrow P X$ such that (i) for each $x \in X, \cap_{n \in \omega} B(n, x)=\{x\}$ and for each $n \in \omega, B(n+1, x) \subset B(n, x)$, and (ii) each set $U \subset X$ is open if and only if for each $x \in U$ there is $n_{x} \in \omega$ with $B\left(n_{x}, x\right) \subset U$.

(a) A space which admits a wfc-system is called weakly first countable (or is said to satisfy the gf axiom of countability) [A].

(b) An $\mathscr{F}$-space [HS] is a space $X$ which admits a wfc-system $B$ such that if $x \in X$ and $F \subset X$ is closed with $x \notin F$, then there is $n \in \omega$ such that for each $y \in B(n, x) \backslash\{x\}$ there exists $n_{y} \in \omega$ such that $\{x, y\} \not \subset \cup_{z \in F} B\left(n_{y}, z\right)$. If $B(n, x)$ is a neighborhood of $x$ for each $n \in \omega$ and $x \in X$, then $X$ is called a neighborhood $\mathcal{F}$-space.

(c) An $\mathscr{F}_{r}$-space [D] is a space $X$ which admits a wfc-system $B$ such that if $x \in X$ and $F \subset X$ is closed with $x \notin F$, then there is $n \in \omega$ such that for each $y \in B(n, x) \backslash\{x\}$ there exists $n_{y} \in \omega$ such that $y \notin \cup_{z \in F} B\left(n_{y}, z\right)$. If $B(n, x)$ is a neighborhood of $x$ for each $n \in \omega$ and $x \in X$, then $X$ is called a neighborhood $\mathcal{F}_{r}$-space.

Received by the editors March 27, 1978 and, in revised form, August 11, 1978.

AMS (MOS) subject classifications (1970). Primary 54D25; Secondary 54E25, 54E30, 54D30.

Key words and phrases. Weakly first countable, $\mathcal{F}_{\text {-space, }} \mathcal{F}_{r}$-space, feebly compact, Baire space. 


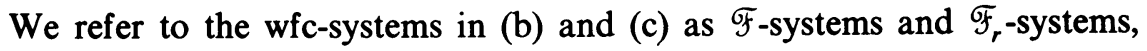
respectively. Studies of properties of spaces of type (a), (b) and (c), are given in [HS], [ $\left.\mathbf{S}_{3}\right]$, [D], and [DGN].

1. Characterization theorems. We first recall that a point $p$ is a $\theta$-adherent point of filterbase $t$ provided that for each $T \in t$ and neighborhood $V$ of $p$, we have $T \cap \bar{V} \neq \varnothing$.

THeORem 1.1a. Let $(X, \tau)$ be a Hausdorff weakly first countable space. The following are equivalent.

(i) The space $(X, \tau)$ is minimal weakly first countable.

(ii) Every countable filterbase on $(X, \tau)$ which has a unique $\theta$-adherent point is convergent.

THEOREM 1.1b. Let $(X, \tau)$ be a Hausdorff $\mathscr{F}$-space. The following are equivalent.

(i) The space $(X, \tau)$ is minimal $\mathscr{F}$.

(ii) Every countable filterbase on $(X, \tau)$ which has a unique $\theta$-adherent point is convergent.

THEOREM 1.1c. Let $(X, \tau)$ be a Hausdorff $\mathscr{F}_{r}$-space. The following are equivalent.

(i) The space $(X, \tau)$ is minimal $\mathscr{F}_{r}$.

(ii) Every countable filterbase on $(X, \tau)$ which has a unique $\theta$-adherent point is convergent.

Proof of 1.1a. Suppose $(X, \sigma)$ is weakly first countable Hausdorff space and $\sigma \subset \tau$. Let $B$ be a wfc-system for $(X, \sigma)$. For each point $p \in X$, the filterbase $\mathscr{B}_{p}=\{B(n, p): n \in \omega\}$ has a unique $\theta$-adherent point in $(X, \sigma)$, namely $p$. Since $\sigma \subset \tau, \mathscr{S}_{p}$ has a unique $\theta$-adherent point in $(X, \tau)$. Hence $\mathscr{B}_{p}$ converges to $p$ in $(X, \tau)$. Now if $T \in \tau$ and $p \in T$, then there exists $n_{p} \in \omega$ such that $B\left(n_{p}, p\right) \subset T$, i.e., $T \in \sigma$. Hence (ii) implies (i).

Conversely, suppose $q \in X$ and $t=\left\{F_{n}: n \in \omega\right\}$ is a filterbase on $X$ such that

(1) for each $n \in \omega, F_{n} \supset F_{n+1}$,

(2) $q$ is the unique $\theta$-adherent point of $t$ in $(X, \tau)$,

(3) $t$ is not convergent, and

(4) $F_{0}=X$.

Let $\sigma=\{V \in \tau: q \in V \Rightarrow$ there is $F \in t$ with $F \subset V\}$. Then it is not difficult to prove that $\sigma$ is strictly weaker than $\tau$ and $(X, \sigma)$ is a Hausdorff weakly first countable space.

Proofs of Theorems $1.1 \mathrm{~b}$ and $1.1 \mathrm{c}$ are similar.

Concerning $P$-closed spaces, one can easily obtain the following. 
THEOREM 1.2a. Let $X$ be a Hausdorff weakly first countable space. The following are equivalent.

(i) $X$ is weakly first countable-closed.

(ii) Every countable filterbase on $X$ has a $\theta$-adherent point.

THEOREM 1.2b. Let $X$ be a Hausdorff $\mathscr{F}$-space. The following are equivalent.

(i) $X$ is $\mathscr{F}$-closed.

(ii) Every countable filterbase on $X$ has a $\theta$-adherent point.

THEOREM 1.2c. Let $X$ be a Hausdorff $\mathscr{F}_{r}$-space. The following are equivalent.

(i) $X$ is $\mathscr{F}_{r}$-closed.

(ii) Every countable filterbase on $X$ has a $\theta$-adherent point.

Proof of 1.2a. Suppose $X$ is weakly first countable, Hausdorff and there exists a weakly first countable Hausdorff space $Y$ with $X \subsetneq \bar{X} \subset Y$. Since $\bar{X}$ is a closed subset of $Y$, the space $\bar{X}$ is a weakly first countable Hausdorff space. Let $B$ be a wfc-system for $\bar{X}$. Since $X$ is not closed in $\bar{X}$, there exists a point $p \in \bar{X} \backslash X$ such that $B(n, p) \cap X \neq \varnothing$ for every $n \in \omega$. Let $F_{n}=$ $B(n, p) \cap X$ for each $n \in \omega$, and let $t=\left\{F_{n}: n \in \omega\right\}$. Then $t$ is a countable filterbase on $X$, and since $Y$ is Hausdorff, $t$ has no $\theta$-adherent point in $X$.

Conversely, suppose $t=\left\{G_{n}: n \in \omega\right\}$ is a filterbase on $X$ such that $G_{0}=$ $X, G_{n} \supset G_{n+1}$ for each $n \in \omega$, and $t$ has no $\theta$-adherent point in $X$. Choose $q \notin X$, and let $Y=X \cup\{q\}$. Call $V \subset Y$ open if and only if $V \cap X$ is open in $X$ and if $q \in V$, then there exists $n \in \omega$ with $G_{n} \subset V$.

One can easily verify that $X$ is a proper dense subspace of $Y$ and that $Y$ has as a wfc-system the function $B^{*}$, where $B^{*}$ is given by

$$
B^{*}(n, x)=\left\{\begin{array}{l}
B(n, x) \text { if } x \in X, \\
G_{n} \cup\{q\} \text { if } x=q, \quad \text { where } n \in \omega .
\end{array}\right.
$$

The proofs of Theorems $1.2 \mathrm{~b}$ and $1.2 \mathrm{c}$ are similar.

For many properties $\mathrm{P}, \boldsymbol{P}$-minimality is sufficient to force $\boldsymbol{P}$-closedness. This is also true of these properties.

COROLlARY 1.2.1a. Every minimal weakly first countable space is weakly first countable-closed.

Corollary 1.2.1b. Every minimal $\mathscr{F}$-space is $\mathscr{F}$-closed.

COROllary 1.2.1c. Every minimal $\mathscr{F}_{r}$-space is $\mathscr{F}_{r}$-closed.

Proof. If $(X, \tau)$ is a $P$-minimal space for $P \in$ \{weakly first countable, $\left.\mathscr{F}_{F}, \mathscr{F}_{r}\right\}$, and $t$ is a descending sequence of nonempty sets having no $\theta$ adherent in $(X, \tau)$, we pick a point $q \in X$. The constructions used in Theorems 1.1 provide a weaker $P$-topology $\sigma$ on $X$.

Example 7 of $\left[\mathbf{S}_{2}\right]$ is a Hausdorff-closed space $Y$ which is semimetrizable but has a weaker metric topology. Hence there is no hope of reversing the implications in Corollaries 1.2.1. 
COROLLARY 1.2.2b. Every regular $\mathscr{F}$-closed space is compact.

COROLLARY 1.2.2c. Every regular $\mathscr{F}_{r}$-closed space is compact.

Proof. For regular spaces, adherence and $\theta$-adherence are equivalent. In

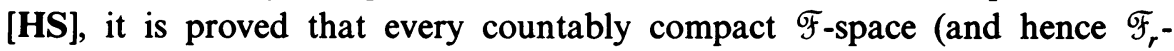
space) is compact.

The assumption of regularity is not superfluous in the above corollary.

Example 6 of $\left[\mathbf{S}_{2}\right]$ is an example due to Urysohn of a noncompact minimal Hausdorff space. Stephenson shows the space to be semimetrizable, hence minimal $\mathscr{F}$, minimal $\mathscr{F}_{r}$ and minimal symmetrizable. Without the assumption of regularity, we have the following results. As in $\left[\mathbf{S}_{2}\right.$, Corollary 5] one can obtain the following.

COROLlaRY 1.2.3b. Let $X$ be a Hausdorff $\mathcal{F}$-space in which every closed subset is $\mathscr{F}$-closed. Then $X$ is compact.

CoROllaRY 1.2.3c. Let $X$ be a Hausdorff $\mathscr{F}_{r}$-space in which every closed subset is $\mathscr{F}_{r}$-closed. Then $X$ is compact.

For first countable spaces, we state several results which can be established by arguments similar to ones given in $[\mathbf{G}]$ and $\left[\mathbf{S}_{\mathbf{1}}\right]$.

Recall that a space is feebly compact if every countable open filterbase has an adherent point. A space is semiregular if it has a base of regular open sets.

THEOREM 1.3b. Let $X$ be a Hausdorff neighborhood $\mathcal{F}_{\text {-space. The following }}$ are equivalent.

(i) $X$ is neighborhood $\mathcal{F}$-closed.

(ii) $X$ is feebly compact.

THEOREM 1.3c. Let $X$ be a Hausdorff neighborhood $\mathscr{F}_{r}$-space. The following are equivalent.

(i) $X$ is neighborhood $\mathcal{F}_{r}$-closed.

(ii) $X$ is feebly compact.

THEOREM 1.4b. Let $X$ be a Hausdorff neighborhood $\mathcal{F}$-space. The following are equivalent.

(i) $X$ is minimal neighborhood $\mathcal{F}$.

(ii) Every countable open filterbase on $X$ with a unique adherent point is convergent.

(iii) $X$ is semiregular and neighborhood $\mathscr{F}$-closed.

THEOREM 1.4c. Let $X$ be a Hausdorff neighborhood $\mathscr{F}_{r}$-space. The following are equivalent.

(i) $X$ is minimal neighborhood $\mathscr{F}_{r}$.

(ii) Every countable open filterbase on $X$ with a unique adherent point is convergent.

(iii) $X$ is semiregular and neighborhood $\mathscr{F}_{r}$-closed. 
2. A density theorem. In this section, we prove a theorem from which we may obtain G. M. Reed's theorem that every Moore-closed space is separable and Stephenson's theorem that every Baire, semimetrizable-closed space is separable.

LEMMA 2.1. Let $X$ be a Baire neighborhood $\mathcal{F}$-space with $B$ an $\mathcal{F}$-system for $X$ such that $B(n, x)$ is open for each $n \in \omega$ and $x \in X$. Suppose $V$ is an open subset of $X$ which contains no isolated points, and for each $m>0$ let $V_{m}=V \backslash$ $\left(\cup_{x \in X \backslash V} B(m, x)\right)$. Then for some $m>0, V_{m}$ has nonempty interior.

Proof. Define $W=V \cap\left(\cap_{m>0}\left(\cup_{x \in X \backslash V} B(m, x)\right)\right)$ and $V_{0}=V \cap \bar{W}$. Now $V=\cup_{i \in \omega} V_{i}$ and since $X$ is Baire, some $V_{i}$ must have nonempty interior. We will prove that $V_{0}$ has empty interior, and the result will follow.

On the contrary, suppose that there exists a nonempty open set $I$ with $I \subset V_{0}$.

Now $W$, with the subspace topology, is a discrete space, for consider any point $x \in W$. Select $n \in \omega$ such that for each $y \in B(n, x) \backslash\{x\}$ there exists $n_{y} \in \omega$ such that $\{x, y\} \not \subset \cup_{z \in X \backslash V} B\left(n_{y}, z\right)$. For each $y \in B(n, x) \backslash\{x\}$, one has $y \notin \cup_{z \in X \backslash V} B\left(n_{y}, z\right)$, since $x \in \cup_{z \in X \backslash V} B\left(n_{y}, z\right)$, and so $B(n, x) \cap W$ $=\{x\}$.

Because $W$ is dense in $\mathrm{Cl}_{V} W=V_{0}$, it follows that each point of $W$ is isolated in the space $V_{0}$. But $I \subset V_{0}$ and $I$ is nonempty and open, so there exists a point $w \in W \cap I$. Now $w$ is an isolated point of the open set $I$, and, hence, is isolated in $X$, but $V$ cannot contain isolated points. This contradiction establishes the result.

THEOREM 2.2. Suppose $X$ is a Baire, feebly compact neighborhood $\mathcal{F}_{\text {-space, }}$ and let $I$ be the set of isolated points of $X$. Then $X$ has dense subset $C$ with $|C| \leqslant \max \left\{|I|, \aleph_{0}\right\}$.

Proof. Let $K=\max \left\{|I|, \aleph_{0}\right\}$, and denote by $B$ an $\mathscr{F}$-system for $X$ with each $B(n, x)$ an open set. We will first prove that for every pairwise disjoint family $\mathcal{V}$ of open subsets of $X$, we have $|\mathcal{V}| \leqslant K$.

Suppose, on the contrary, that there exists an open disjoint collection $\mathcal{T}$ with $|\mathcal{T}|>K$. There is an uncountable subset $\mathcal{V}$ of $\mathcal{T}$ such that each $V \in \mathcal{V}$ is nonempty and contains no isolated points. For each $V \in \mathcal{V}$ and $m \in$ $N(=\omega \backslash\{0\})$, let $V_{m}=V \backslash\left(\cup_{x \in X \backslash V} B(m, x)\right)$. By Lemma 2.1, for each $V \in \mathcal{V}$, we may choose $m(V) \in N$ such that $V_{m(V)}$ has nonempty interior. Choose $k \in N$ such that $\mathscr{W}=\{V \in \mathcal{V}: m(V)=k\}$ is uncountable and for $W \in \mathscr{W}$, let $J_{W}$ denote the interior of $W_{k}$. By the feeble compactness of $X$, there is a point $x \in X$ at which $\left\{J_{W}: W \in \mathscr{W}\right\}$ fails to be locally finite. For each $W \in \mathcal{W}$, if $B(k, x) \cap J_{W} \neq \varnothing$, then $x \in W$. However, $\{W \in \mathcal{W}$ : $\left.B(k, x) \cap J_{W} \neq \varnothing\right\}$ is infinite. This is a contradiction to the disjointness of थ.

Now let us construct $C$. For each $n \in N$, let $\mathscr{B}_{n}=\{B(k, x): x \in X$ and 
$k \geqslant n\}$, and let $\mathscr{D}_{n}$ be a maximal disjoint subcollection of $\mathscr{B}_{n}$, such that $\{y\} \in \mathscr{D}_{n}$ for each $y \in I$. For each $n \in N$ and $D \in \mathscr{D}_{n}$ choose $x(n, D)$ such that for some $k \geqslant n, D=B(k, x(n, D))$, and let $C_{n}=\left\{x(n, D): D \in \mathscr{D}_{n}\right\}$. Let $C=\cup_{n \in N} C_{n}$. Then $\left|C_{n}\right| \leqslant K$ for each $n \in N$, so $|C| \leqslant K$.

For each $n \in N, \cup \mathscr{D}_{n}$ is dense and open, so $E=\bigcap_{n \in N}\left(\cup \mathscr{D}_{n}\right)$ is dense in $X$. To show that $\bar{C}=X$, it suffices for us to prove $E \subset \bar{C}$.

Suppose $x \in E \backslash \bar{C}$. Choose $n \in N$ such that for each $y \in B(n, x) \backslash\{x\}$, there exists $n_{y} \in N$ with $\{x, y\} \not \subset \cup_{z \in \bar{C}} B\left(n_{y}, z\right)$. Then since $x \notin I$, there is a point $y \in(B(n, x) \backslash\{x\}) \cap E$, but then $\{x, y\} \subset E=\bigcap_{n \in N}\left(\cup \mathscr{D}_{n}\right) \subset$ $\cup \mathscr{D}_{n_{y}} \subset \cup_{z \in \bar{C}} B\left(n_{y}, z\right)$, a contradiction.

COROLlARY 2.2.1. Every Baire, feebly compact, neighborhood $\mathcal{F}$-space with no more than countably many isolated points is separable.

COROLlARY 2.2.2 [ $\left.\mathbf{S}_{2}\right]$. Every Baire, feebly compact, semimetrizable space is separable.

COROLlaRY 2.2.3 [R]. Every Moore-closed space is separable.

Corollary 2.2.1 is obvious from the theorem. To see how Corollaries 2.2.2 and 2.2.3 follow, we first recall $\left[\mathbf{S}_{3}\right.$, Theorem 17] that a feebly compact symmetrizable space can have at most countably many isolated points. Next, every Moore closed space is feebly compact, Baire and semimetrizable. Finally, of course, every semimetrizable space is a neighborhood $\mathscr{F}$-space.

It is known [M] that every regular feebly compact space is Baire. Hence we have the following restatement of Corollary 2.2.1 for the regular case.

COROLlaRY 2.2.4. Every regular neighborhood $\mathcal{F}$-closed space with no more than countably many isolated points is separable.

It is impossible to remove the first countability from the last theorem. Stephenson has recently given an example of a feebly compact, Baire, symmetrizable Hausdorff space which is not separable $\left[\mathbf{S}_{\mathbf{4}}\right]$. Also, the consideration of the isolated points can not be eliminated. The Aleksandrov double interval is a compact, Hausdorff, Baire, neighborhood $\mathscr{F}_{r}$-space which is not separable.

The question now naturally arises, "Can you restrict the isolated points by the cardinality of the continuum?" (denoted by $c$ ). We provide the following partial solution.

THEOREM 2.3. Let $X$ be a Hausdorff feebly compact $\mathscr{F}_{r}$-space and suppose that for each point $x \in X$, there is an open neighborhood $V_{x}$ of $x$ with $\left|V_{x}\right| \leqslant c$. Then $X$ has at most $c$ isolated points. 
Proof. Let $I$ be the set of isolated points of $X$ and suppose that $|I|>c$. Let $\mathfrak{N}$ be a maximal open disjoint collection of subsets of $X$ such that for each $M \in \Re,|M| \leqslant c$ and $M \cap I^{\prime} \neq \varnothing$. We will prove that $|\Re|>c$.

Suppose that $|\mathfrak{N}| \leqslant c$. Then $|\cup \mathfrak{R}| \leqslant c$ and since $X$ is a sequential Hausdorff space, the set $K=\mathrm{Cl}(\cup \mathfrak{K})$ has $|K| \leqslant c$. Thus we can construct an open set $V$ with $|V| \leqslant c$ and $K \subset V$. Then $|\bar{V}| \leqslant c$, so there is an infinite set $J$ of isolated points of $X$ with $J \subset X \backslash \bar{V}$. By the feeble compactness of $X$, there exists a point $y$ which is a limit point of $J$. Since necessarily $y \notin V$ and $K \subset V$ we can find an open neighborhood $W$ of $y$ with $|W|<c$ and $W \cap K=\varnothing$. But then $\mathfrak{K}^{\prime}=\mathfrak{K} \cup\{W\}$ would contradict the maximality of $\Re$.

Thus, $\mathfrak{N}$ is, in any case, uncountable. Let $Y=X \backslash(\cup \mathfrak{T})$. Let $B$ be an $\mathscr{F}_{r}$-system for $X$. Using the fact that $\mathscr{N}$ is pairwise disjoint and each $M \in \mathfrak{N}$ contains a point of $X \backslash I$, one can find an integer $k$ and an infinite subset $T$ of $I$ such that $T \cap\left(\cup_{y \in Y} B(k, y)\right)=\varnothing$ and $|T \cap M| \leqslant 1$ for each $M \in \mathfrak{R}$. Thus, for each point $x \in X \backslash T$, there exists $n \in \omega$ with $B(n, x) \cap$ $T=\varnothing$, from which it follows that $T$ is a closed subset of $X$. This latter, however, would be a contradiction of the feeble compactness of $X$.

COROllary 2.3.1. Let $X$ be a locally compact Hausdorff, feebly compact $\mathscr{F}_{r}$-space. Then $X$ has cardinality $\leqslant c$.

Results of Jean Larson, George McNulty and Peter Harley show that Hausdorff may not be omitted from Theorem 2.3. They have shown that any infinite cardinal $k$ with the cofinite topology is symmetrizable. The double of this space would be a compact $\mathscr{F}_{r}$-space with $k$ isolated points.

\section{REFERENCES}

[A] A. V. Arhangel'skii, Mappings and spaces, Russian Math. Surveys 21 (1966), 115-162.

[C] Douglas E. Cameron, $A$ survey of maximal topological spaces, Eleventh Annual Spring Topology Conference, Baton Rouge, Louisiana, 1977.

[D] S. W. Davis, On $\mathscr{F}_{r}$-spaces, General Topology and Appl. 9 (1978), 131-138.

[DGN] S. W. Davis, G. Gruenhage and P. J. Nyikos, $G_{\delta}$-sets in symmetrizable and related spaces, General Topology and Appl. 9 (1978), 253-261.

[G] J. W. Green, Moore-closed spaces, completeness, and centered bases, General Topology and Appl. 4 (1974), 297-313.

[HS] Peter W. Harley III and R. M. Stephenson, Jr., Symmetrizable and related spaces, Trans. Amer. Math. Soc. 219 (1976), 89-111.

[M] R. A. McCoy, A filter characterization of regular Baire spaces, Proc. Amer. Math. Soc. 40 (1973), 268-270.

[R] G. M. Reed, On chain conditions in Moore spaces, General Topology and Appl. 4 (1974), 255-267.

[S, R. M. Stephenson, Jr., Minimal first countable topologies, Trans. Amer. Math. Soc. 138 (1969), 115-127.

$\left[\mathrm{S}_{2}\right]$ _ Symmetrizable-closed spaces, Pacific J. Math. 68 (1977), 507-514.

$\left[\mathrm{S}_{3}\right] \ldots$, Symmetrizable, $\mathcal{F}_{-}$, and weakly first countable spaces, Canad. J. Math. 29 (1977), $480-488$.

[S $\left.\mathbf{S}_{4}\right]$ Near compactness and separability of symmetrizable spaces, Proc. Amer. Math. Soc. 68 (1978), 108-110. 
S. W. DAVIS AND R. M. STEPHENSON, JR.

[W] Stephen Willard, General topology, Addison-Wesley, Reading, Mass., 1970.

Department of Mathematics, Auburn University, Auburn, Alabama 36830

Department of Mathematics, Minm University, Oxpord, Oho 45056 (Current address of S. W. Davis)

Department of Mathematics, Computer Science, and Statistics, University of SOUTH Carolina, Columbia, South Carolina 29208 (Current address of R. M. Stephenson, Jr.) 